\title{
A case of atrioventricular junction ablation and permanent left bundle branch pacing for patient with refractory atrial fibrillation and hypertrophic cardiomyopathy
}

\author{
Ning Zhang ${ }^{1}$, Shan Liu ${ }^{1}$, Shou Zhang ${ }^{1}$, Yan Wei ${ }^{1}$, Le Xie ${ }^{2}$, and Xin $\mathrm{Li}^{1}$ \\ ${ }^{1}$ Linyi People's Hospital \\ ${ }^{2}$ Shandong Medical College
}

November 26, 2020

\begin{abstract}
Atrioventricular node ablation (AVNA) combined with His bundle pacing (HBP) are feasible, safe, and effective in patients with refractory atrial fibrillation (AF), however, the pacing parameters of sensing and capture threshold maybe sometimes unsatisfactory. Left bundle branch pacing (LBBP) provides obvious advantage in patients with conduction diseases at the distal His bundle for its better sensing, a lower and more stable capture threshold. Among hypertrophic cardiomyopathy (HCM) patients, AF is a common sustained arrhythmia, primarily caused by left atrial dilatation and remodeling. Few is known about the feasibility of electrophysiological performance, safety and clinical effectiveness of atrioventricular junction ablation (AVJA) combined with LBBP in patient with refractory AF and HCM. Here, we report a case of a 56-year-old woman suffering from refractory AF and HCM, however HBP was failed for its unsatisfactory sensing, a high and unstable capture threshold for her, therefore, ablation and LBBB were accepted by her to achieve better rate control. Improvement in symptoms, quality of life, and exercise capacity has been observed during the 1.5-year follow-up. To our knowledge, our case originally confirmed that the combination of AVJA and LBBP, without the defect of AVNA combined with HBP, is a better strategy with feasibility and safety for refractory AF patients with comorbidity of HCM, additionally, it may make LBBP more applicable and valuable among patients suffering from HCM meanwhile pace maker treatments are essential.
\end{abstract}

A case of atrioventricular junction ablation and permanent left bundle branch pacing for patient with refractory atrial fibrillation and hypertrophic cardiomyopathy

Ning Zhang ${ }^{1 *}$, Shanwen Liu ${ }^{2 *}$, Shouwen Zhang ${ }^{1}$, Yanjin $\mathrm{Wei}^{1}$, Le $\mathrm{Xie}^{3}$, Xinhua $\mathrm{Li}^{1}$,

1 Department of Cardiology, Linyi People's Hospital, Linyi, Shandong 276000, P.R. China;

2 Department of ECG diagnosis center, Linyi People's Hospital, Linyi, Shandong 276000, P.R. China;

3 Department of Laboratory Medicine, Shandong Medical College, Linyi, Shandong 276000, P.R. China,

* These authors share first authorship on this work.

Funding: None.

Disclosures: None.

Conflict of interests: All the authors declare that there are no conflict of interests.

Corresponding Author: Dr. Xinhua Li, email: dr.lixinhua@139.com.

\section{[Abstract]}


Atrioventricular node ablation (AVNA) combined with His bundle pacing (HBP) are feasible, safe, and effective in patients with refractory atrial fibrillation (AF), however, the pacing parameters of sensing and capture threshold maybe sometimes unsatisfactory. Left bundle branch pacing (LBBP) provides obvious advantage in patients with conduction diseases at the distal His bundle for its better sensing, a lower and more stable capture threshold. Among hypertrophic cardiomyopathy (HCM) patients, AF is a common sustained arrhythmia, primarily caused by left atrial dilatation and remodeling. Few is known about the feasibility of electrophysiological performance, safety and clinical effectiveness of atrioventricular junction ablation (AVJA) combined with LBBP in patient with refractory AF and HCM. Here, we report a case of a 56-year-old woman suffering from refractory AF and HCM, however HBP was failed for its unsatisfactory sensing, a high and unstable capture threshold for her, therefore, ablation and LBBB were accepted by her to achieve better rate control. Improvement in symptoms, quality of life, and exercise capacity has been observed during the 1.5-year follow-up. To our knowledge, our case originally confirmed that the combination of AVJA and LBBP, without the defect of AVNA combined with HBP, is a better strategy with feasibility and safety for refractory AF patients with comorbidity of HCM, additionally, it may make LBBP more applicable and valuable among patients suffering from HCM meanwhile pace maker treatments are essential.

\section{[Key word]}

Atrioventricular junction ablation; Left bundle branch pacing; Refractory atrial fibrillation; Hypertrophic cardiomyopathy

\section{[Introduction]}

HBP is a more physiologic modality of pacing, a feasible and effective form for ventricular activation, however, the recognition of imperfection of $\mathrm{HBP}$ has led to seek for more advisable options of pacing. Permanent LBBP might be an alternative to HBP to potentially maintain left ventricular electrical synchrony with better sensing, a lower and more stable capture threshold $[\mathbf{1 , 2 , 3}]$, especially in patients with infra-nodal atrioventricular block and left bundle branch block.

Atrioventricular junction region includes atrioventricular node and nearby proximal His bundle, AVNA combined with HBP are feasible, safe, and effective in patients with refractory AF [4], however, successful case of atrioventricular junction ablation (AVJA) combined with LBBP in refractory AF with comorbidity of HCM has not been documented.

Our report exactly focuses on the feasibility of electrophysiological performance, safety and clinical effectiveness of AVJA combined with LBBP in patient with refractory AF and HCM.

\section{[Case summary]}

A 56-year-old woman had presented with symptoms of paroxysmal palpitation and chest tightness for 6 almost years, was admitted to our hospital this time with 10-day recurrence.

An operation of percutaneous mitral balloon dilation and valvuloplasty was performed on her for severe mitral stenosis caused by rheumatic heart disease at 13 years ago, and she recovered well postoperatively.

Symptomatic paroxysmal AF started with an episode of several hours at 6 years ago, oral medical therapy of warfarin was started for the subsequently frequent seizures although seizures could be reverted spontaneously. However, the medicine was terminated by herself 6 months later without recommendation of doctor. Cerebral infarction occurred at the fourth day of medical termination with the consequence of right limb hemiplegia, fortunately, medication and rehabilitation therapy were given positively and followed by favorable recovery. The diagnosis of hypertrophic cardiomyopathy (non-obstructive type) was established, while the attempt of radiofrequency ablation of AF was conducted at 3 years ago and failed due to recurrence 6 months later.

2 months ahead of her hospital admission this time, while accompanying her family in the infusion room of our hospital, the patient herself suffered from a sudden syncope along with limbs twitching which was immediately identified by electrocardiogram (ECG) monitoring to be ventricular fibrillation, luckily the sinus rhythm restored by emergency electric defibrillation. 
Her hospitalization this time was due to 10-day recurrence of persistent AF with clinical manifestations of palpitation, chest tightness and dizziness. Key physical examinations included a blood pressure of 108 / $68 \mathrm{mmHg}$, a conscious mind, no moist crackles or wheezes within lungs, absolutely irregular heart rhythm, no extra cardiac sounds or obvious cardiac murmurs, no edema in both lower limbs. ECG showed atrial fibrillation and tachycardia, with mean heart rate of $140 \mathrm{bpm}$. echocardiographic measurements included left atrium diameter of $46 \mathrm{~mm}$, left ventricular end-diastolic diameter of $42 \mathrm{~mm}$, ventricular septal thickness of $21 \mathrm{~mm}$, posterior left ventricular wall thickness of $9 \mathrm{~mm}$, left ventricular ejection fraction of $57 \%$.

\section{[Treatment course]}

Essential strategies were taken for her, however, she failed to revert to sinus rhythm with a reduction of heart rate to about $80 \mathrm{bpm}$, until the tenth day of treatment, she reverted to sinus rhythm which was unable to maintain and with a heart rate of $44 \mathrm{bpm}$. Considering the refractory AF and history of electric defibrillation, therefore, she accepted therapy of AVJA combined with LBBP and implantation of defibrillation electrodes.

\section{[Procedural details]}

\section{[Implantation]}

The left axillary vein was punctured to obtain venous access, an incision was made $4 \mathrm{~cm}$ below the left clavicle to make a capsular bag. the C315 HIS sheath was advanced over a long guide-wire towards the tricuspid annulus, the 3830 lead was then advanced to the tip of the sheath towards His bundle region, HB region was mapped in the unipolar configuration. His potential can be detected[Fig1], His lead was fixed by sightly screw followed by HBP, however HBP showed a unsatisfactory sensing, a high and unstable capture threshold, then His lead was used as a protective lead in case of temporary pacing, and the X-ray reference image of location of His lead was routinely regard as a marker for LBBP lead implantation.

Another 3830 lead was advanced to tip of the sheath towards surface of right ventricular septum, the initial site for LBBP, was approximately $1 \mathrm{~cm}$ distal to the HBP lead position on the surface of right ventricular septum along the line between HBP site and right ventricular apex in the fluoroscopy view of right anterior oblique $\left(\mathrm{RAO} 30^{\circ}\right)$ [Fig1] , At this site, the paced QRS complex morphology usually demonstrates a "w" pattern with a notch at the nadir of the QRS in lead V1 [Fig1][5] .

Then, the sheath was rotated anticlockwise to maintain the orientation of lead tip perpendicular to septal surface and provide adequate support to screw the lead into the septum. As lead tip screwing into interventricular septum, lead parameters ( $R$ wave sensing and LBB capture morphologies and thresholds) were assessed synchronously in both unipolar and bipolar configurations. The following signs will appear: 1) the notch of paced QRS complex in lead V1 will move from nadir to the end [Fig1] . 2) First increase then reduction of pacing impedance in unipolar. 3) the position of lead tip advances with fulcrum sign left behind on fluoroscopy [Fig1] .

The lead tip advanced cautiously until satisfactory parameters were achieved, the latter included lead tip impedance $560 \Omega$, ring impedance $650 \Omega$, bipolar impedance $735 \Omega$, threshold $0.7 \mathrm{~V}$, QRS amplitude $28.5 \mathrm{mV}$, left branch bundle potential cannot be detected during the procedure.

The right ventricular defibrillation lead 6947 was implanted in right ventricle (RV) apex in a standard fashion (rotation, fixation et al.). Parameters of RV lead were identified with threshold $0.7 \mathrm{v}$, the QRS amplitude $5.4 \mathrm{mV}$, and impedance $399 \Omega$.

The system (C315 HIS sheath and 3830 lead) located in His bundle region was then withdrawn to inferior right atrial septum. Parameters of right atrial lead were assessed with threshold $0.4 \mathrm{~V}, \mathrm{P}$ amplitude $2.7 \mathrm{mV}$, and impedance $574 \Omega$.

\section{[Ablation]}

A L8.5 Swartz sheath was delivered through the right femoral vein access, a saline-irrigated ablation catheter was delivered to the proximity of His bundle via the sheath [Fig2]. Ablation was initiated from proximity 
of His bundle with a set of $30 \mathrm{~W}, 43$, and a flow rate of $17 \mathrm{ml} /$ min until complete atrioventricular block indicated the success of ablation [Fig2] . Further lesions were consolidated for $120 \mathrm{~s}(43 ; 30 \mathrm{~W})$ without complications then followed by withdraw of catheter.

Finally, fixed the pacing lead, connected leads to relative channels, the left ventricular pacing was programmed to $80 \mathrm{~ms}$ ahead of RV. The total X-ray exposure time was 16 minutes with no occurrence of complications. The outline for the procedure is shown in Table1[Table 1] .

\section{[Follow-up]}

6-month follow-up showed safe and stable output of pacing with atrial pacing rate (AP) of $83.3 \%$, ventricular pacing rate (VP) of $100 \%$, atrium impedance $551 \Omega$ and sensing $3.1 \mathrm{mV}$, left ventricle impedance $304 \Omega$, right ventricle (RV) impedance 418 Rand sensing $5.4 \mathrm{mV}$, no change of ECG or echocardiogram features [Fig3] .

1.5-year follow-up showed disappearance of previous symptoms with no recurrence, improvement of quality of life and exercise capacity, high patient satisfaction.

There were no adverse events associated with the ablation and implantation procedure, device operation or disease.

\section{[Discussion]}

Satisfactory long-term management of atrial fibrillation is seldom achievable ,fortunately, AVNA combined with permanent pacing therapy is practically confirmed to significantly reduce cardiac symptoms while improving quality of life [6], owing to the well-established safety and efficacy of this proficient technique[7] , AVNA and permanent pacing is regards as IIa class of recommendation (evidence level: B) to control heart rate when pharmacological therapy is inadequate and rhythm control is not achievable [8], they are suggested to be a valuable option in patients with refractory atrial arrhythmia resistant to other treatment modalities [9] .

Among HCM patients, AF is primarily caused by left atrial dilatation and remodeling as a common sustained arrhythmia, conversely, AF can contribute to progressive decline of cardiac function, worsen heart failure and increase risk of systemic thromboembolism [10]. Recent years, LBBP provides significant advantage in patients with conduction disease at the distal His bundle, LBBP emerges for its better sensing , a lower and more stable capture threshold $[\mathbf{1 , 2 , 3}]$, however, implantation indications of this novel technique require further clinical investigation, the role of AVJA combined with LBBP remains unknown in refractory AF and HCM cohorts, whereas safety and feasibility of AVNA combined with HBP have been proven in patients with AF and difficulty in rate control by studies of Vijayaraman P. et al.[4] .

In this case, the rapid ventricular response to $\mathrm{AF}$ did not respond promptly to pharmacological therapies and contributed to ongoing symptoms, furthermore, this patient had a history of recurrence of AF after radiofrequency ablation, therefore, ablation and pacing were accepted by her to achieve better rate control. Implantation of ICD was reasonable for high risk of sudden death induced by atrial tachyarrhythmia or HCM, besides her conclusive history of ventricular fibrillation [11] . By 1.5-year follow-up of this case, AVJN and LBBP strategy is confirmedly associated with improvement in symptoms, quality of life, and exercise capacity. Feasibility and safety of AVJA combined with LBBP for refractory AF patients with HCM, was concluded by this case firstly. The situation of refractory AF patients may become worse if they suffer from HCM meanwhile, because both diseases can worsen the symptomatic condition of each other, lead to the increasement of possibility of sudden death and response poorly to drug treatments, obviously, the combination strategy is a better option, a more reasonable and efficient treatment than AVNA combined with HBP for these patients.

In addition, our success may wider the application in HCM patients for whom pacing therapy is reasonable. The hypertrophy interventricular septum may make the manoeuvr of pacemaker implantation more challenging, our report may provide a valuable reference for similar patients. During the operation of implantation of pacemaker, the lead was moved approximately $1 \mathrm{~cm}$ distally towards the RV apex, followed by deeply 
screwing the lead helix into the hypertrophy interventricular septum, this experience of LBBP for HCM patient is also in line with that from Huang $\mathrm{W}$.et al.[5] .Conventionally, ablation of the atrioventricular node may produce a slightly faster, more stable escape rhythm derived from the His bundle with a narrow QRS complex, whereas ablation lower at the His bundle is likely to produce a slower, broad QRS complex which may be less reliable. However, the total time of performance is longed given that the pacemaker and ICD have to be implanted besides the ablation, after achievement of pacemaker implantation which serves as a safeguard of ablation, the proximity of His bundle was chosen as the target of ablation instead of atrioventricular node in order to reduce total performance time.

Undoubtedly, long-term follow-up and more such cases are needed for further investigation to evaluate the benefits and safety of AVJA combined with LBBP strategy.

\section{[Reference]}

[1] Huang W, Su L, Wu S, et al. A Novel Pacing Strategy With Low and Stable Output: Pacing the Left Bundle Branch Immediately Beyond the Conduction Block. Can J Cardiol Dec. 2017; 33:1736 e1731-1736 e1733.

[2] Vijayaraman P, Subzposh FA, Naperkowski A, et al. Prospective Evaluation of Feasibility, Electrophysiologic and Echocardiographic Characteristics of Left Bundle Branch Area Pacing. Heart Rhythm 2019. DOI: https://doi.org/10.1016/j.hrthm.2019.05.011

[3] Li Y, Chen K, Dai Y, etal. Left bundle branch pacing for symptomatic bradycardia: Implant success rate, safety, and pacing characteristics. Heart Rhythm 2019; DOI: 10. 1016/ j. hrthm. 2019. 05. 014.

[4] Vijayaraman P, Subzposh FA. Atrioventricular node ablation and His bundle pacing. Europace 2017;19: iv10-iv16.DOI:10.1093/ europace /eux263.

[5] Huang W, Chen X, Su L, et al. A beginner's guide to permanent left bundle branch pacing, Heart Rhythm (2019), doi: https://doi.org/10.1016/ j. hrthm. 2019. 06.016.

[6] Wood MA, Brown-Mahoney C, Kay GN. Clinical outcomes after ablation and pacing therapy for atrial fibrillation: a meta-analysis. Circulation 2000; 101:1138-1144.

[7] Lim KT,Davis MJ,Powell A,Arnolda L,Moulden K,Weerasooriya R,Ablate and pace strategy for atrial fibrillation:long-term outcome of AIR-CRAFT trial. Europace 2007;9(7):498-505

[8] January CT, Wann LS, Alpert JS, et al.2014 AHA/ACC/HRS guideline for the management of patients with atrial fibrillation: executive summary. Circulation 2014;130(23):2071-2104.

[9] Kevin Willy. Florian Reinke. Christian Ellermann, et al. Long-term experience of atrioventricular node ablation in patients with refractory atrial arrhythmias. Heart and Vessels (2019). https://doi.org/10.1007/s00380-019-01536-5.

[10] Lohit Garg, Manasvi Gupta, Syed Rafay Ali Sabzwari, et al. Atrial fibrillation in hypertrophic cardiomyopathy: prevalence, clinical impact, and management. Heart Failure Reviews (2018), doi: https://doi.org/10.1007/s10741-018-9752.

[11] Epstein AE, Di Marco JP, Ellenbogen KA, et al.2012 ACCF/AHA/HRS focused update incorporated into the ACCF/AHA/HRS 2008 guidelines for device-based therapy of cardiac rhythm abnormalities: a report of the American College of Cardiology Foundation/American Heart Association Task Force on Practice Guidelines and the Heart Rhythm Society. J Am Coll Cardiol 2013; 61: e6-75. DOI: 10.1016/j.jacc.2012.11.007.

\section{Hosted file}

Figures.pdf available at https://authorea.com/users/379023/articles/495398-a-case-ofatrioventricular-junction-ablation-and-permanent-left-bundle-branch-pacing-for-patientwith-refractory-atrial-fibrillation-and-hypertrophic-cardiomyopathy 


\section{Hosted file}

Table.pdf available at https://authorea.com/users/379023/articles/495398-a-case-ofatrioventricular-junction-ablation-and-permanent-left-bundle-branch-pacing-for-patientwith-refractory-atrial-fibrillation-and-hypertrophic-cardiomyopathy 